\title{
AN EFFICIENT LOSSLESS MEDICAL IMAGE COMPRESSION TECHNIQUE FOR TELEMEDICINE APPLICATIONS
}

\author{
Shivaputra ${ }^{1}$, H.S.Sheshadri ${ }^{2}$, V.Lokesha ${ }^{3}$ \\ ${ }^{1}$ Department of ECE, Assistant Professor, Dr Ambedkar Institute of Technology,India \\ and Research scholar @ Jain University, India. \\ ${ }^{2}$ Department of ECE, Professor and Research Dean, PES College of Engineering,India \\ ${ }^{3}$ Department of Mathematics, Associate Professor, Vijayanagara Sri Krishnadevaraya \\ University,India
}

\begin{abstract}
"If your compassion does not include yourself, it is incomplete" - Gautama Buddha

Telemedicine; use of telecommunication and information technological services, which permits the communication between the users with convenience and fidelity, as well transmitting medical, images and health informatics data. Numerous image processing applications like Satellite Imaging, Medical Imaging and Video has images with too large size or stream size, with a large amount of space or high bandwidth for communication in its original form. Integrity of the transmitted medical images and the informatics data, without any compromise in the data is an essential product of telecommunication and information technology. A colossal need for an adequate compression methodology, in adoption for the compression of medical images /data, to domicile for various metrics like high bandwidth, resolution factors, storage of the images/data, the obligation to perpetuate the validity and precision of data for subsequent perceived diagnosis transactions. This leverages exacting coercions on the restoration error. In this paper we survey the literature related to the Image Processing Methodologies based on ROI technique/s for Digital Imaging and Communication for Medicine (DICOM). A scrutiny as such persuades with the several congestions related to prospective techniques of lossless compression, recommending for a better and a unique image compression technique.
\end{abstract}

\section{Key Words}

ROI (Region of Interest), DICOM (Digital Imaging and Communication for Medicine), Telemedicine, Lossy \& Lossless Compression Techniques.

\section{Introduction}

"A nation is a society united by a delusion about its ancestry and by common hatred of its neighbours" - William R Inge

The comprehensive aspects of compression methodologies for medical images/data, considered among the variants are as follows; 
- High Lossless Compression Ratios

- Resolution Scalability: ability to scale or decode the compressed image at various image resolutions

- Quality Scalability: ability to scale or decode the compressed images at various qualities/SNR etc.

- Image Data Integrity

- Transmission Integrity/Security

- Storage Conditions.

The study of such medical images/data has proven to be an emerging field with an ascend in divergent services/applications related to telehealth, biomedicine, and various other telemedical analyses. The conducive amount of data embedded in these medically produced images from various procedures among which PET/CT, MRI, Bone Densitometry, Ultrasound and other medical related scans. These fields produce images which require more space for storage, the management of which becomes very difficult. These images also demand for high end networks for their transmission such as in Telemedicine application. In the meantime, the compression techniques are classified into lossless and lossy compression techniques. Lossy Image Compression schemes are not generally used in telemedicine applications, due to possible loss of useful clinical information which adversely affect the diagnosis. Thus the need for a Lossless Image Compression scheme pitches in addressing the above issues and as well the storage of the medical images, which requires the best possible quality image to be stored.

DICOM, an ideal standard solution pioneered for superintending, caching, composing and transmitting data pertaining to those medical images, which also includes characterization of the file pattern for castling of the data pertaining to those images between the singletons, capable of acquiring the data as well as the image of the same, and a pact in the grid using suitable TCP/IP between the systems to exchange the these file formats. Thus for the application of telemedicine a suitable DICOM file has to encoded, transmitted and decoded at the receiving end. It is thus required to have a efficient algorithm that accepts the DICOM file encodes at the transmitting end, transmits the file with a suitable security clause and receive the DICOM file and decode the same.

\subsection{DICOM (Digital Imaging and Communication in Medicines)}

During the early 1970s computed Tomography was introduced as the first digital modality in the field of digital medical image processing, the importance of which had increased acquired in the due course of time. The evolutionary aspect of PACS and its distribution to a medical centre or a hospital, electronically, has created the substantiating demand for the exchange of these digital medical images between and among various medical devices from different manufacturers. It was during 1983, both ACR and NEMA forged to create a high-functioning group to develop an image exchange standard. The collective work resulted in ACR-NEMA standard, which was revised several times. Inspite of various revisions, there were certain conceptual weaknesses like no network support for transmission, different proprietary dialects, this standard was however a no success. As a consequence, DICOM standard was developed with an objective to create an open source platform(vendor independent) for the communication of medical images and related data. Since then the DICOM is accepted as a formal standard. The metrics, data values and the 
information contained in an image of DICOM standard do well above the standard definition, defining the format of commerce for medical images defining;

- Data Structures

- Network Oriented Services

- Formats for Storage Media Exchange

- Requirements for conforming Devices and Programs.

Each tone of medical imaging is squarely defined by and pro-founded by the DICOM standard with a well established the grid privileges with a perception of the client-server composition. The network services application agree on a common set of parameters, the connection then can be and will be established.

In medicine, DICOM has developed into an imperative integral for the assimilation of many digital imaging systems. An overture of solutions, for many grid related pertinences as well as offline services are provided by DICOM. There is however no guarantee for a "plug and play" integration of all information systems in a hospital.

\subsection{ROI (Region Of Interest)}

A subspace of the pixels/pixel values pertaining to the input digital image are refined in most of the image akin undertakings. A capricious sector of selected pixels or only a legitimate of the input image, may be considered depending on the task. Due to certain limitations fencing around the compression algorithms; either lossy or lossless, the basic concept of Region of Interest was introduced. ROI, a glossary pattern often correlated with unequivocal or perceptible information encompassing an image, expressed in a structured format. In a medical image, certain regions are of high importance, the data of these sections are to be maintained. Hence an efficient and a practical methodology is required to be modeled in order to retain the information without any loss in the image data.

\section{Existing Systems}

Most of the medical images has three sections ROI (diagnostically important section), Non-ROI, Background (the non image section). The retrospective results achieved through the compression of an image in majority of the existing systems pursue the craft in observing an image, reckoning the ROI of the image, application of lossless or a lossy compression technique to achieve the result. Depending on the selected parts by the radiologists the ROI mask for a medical image is selected in such a way that the pixel values in the background are made zero while the foreground is totally included. After the masking, the two separate parts are segregated as per the ROI and the Non-ROI sections and the lossless compression technique will be applied to the ROI section while the lossy compression technique for the latter section [1]. Thus obtained compressed image progressively transmitted over a secured TCP/IP network. ROI section is compressed with the lossless techniques such as Huffman, LZW, ZIP, RLE, etc, while the Non-ROI is compressed using SPIHT Algorithm after the wavelet transform [1], which offers multi resolution capabilities. 


\subsection{Integer Wavelet Transform}

The Integer Wavelet Transform (IWT) is used to have lossless processing. The wavelet transform (WT), in general, produces floating point coefficients. Although these coefficients can be used to reconstruct an original image perfectly in theory, the use of finite precision arithmetic and quantization results in a lossy scheme. Recently reversible integer wavelet transform has been introduced. Lifting provides an efficient way to implement the Discrete Wavelet Transform (DWT) and the computational efficiency of the lifting implementation can be up to $100 \%$ higher than the traditional direct convolution based implementation (Calderbank et al 1998). Lifting allows simple inverse transform of the same complexity as the forward one. Reversible IWT is composed of the elementary operations of the forward one, taken in reverse order (Reichel et al 2001).

The advantages of IWT are

- Faster calculation with respect to traditional DWT.

- An ephemeral memory is not required as the calculation of the transforms are done wholly.

- An integer value is spawned, whose gauging intricacy is low as compared to DWT, generating floating point numbers.

- Completely reversible, with zero practical loss.

\section{Proposed System}

On a contrary note with the precision in the error value permits given by DWT, an endorsement of IWT is being made, considering the tract of reconstructing the input image. Utmost images accommodate superfluous information, needed to be classified by the user to seize upon the process of compression. In a varsity of censuring medical applications, recommendation of IWT highlights its perfect reconstruction equity. The consequences are exceptional with the ROI-based compression techniques, on contrary with various other lossless methods, with sustentation of diagnostically significant information.

In this proposal we have various differentiated steps revolving around the proposal of an efficient algorithm for compression, progressive transmission over a network and analysis for the errors based on a predictor equation for each sub band of the image compression;

$\checkmark$ A DICOM file/medical image from one of the above said modalities are taken in by the algorithm

$\checkmark \quad$ The DICOM file considered is segmented using the Threshold factor.

$\checkmark$ Then the nominal classification of the ROI, Non-ROI and the background sections is done by the algorithm.

$\checkmark$ Parameters for the ROI selection are based upon the constraints set so the algorithm considers the ROI automatically

$\checkmark$ Lossless Compression Techniques for both the ROI and the Non-ROI sections are applied on a contrary to the work proposed in [1]. 
$\checkmark$ The processed DICOM file is then compressed with the transformation using the IWT Decomposition and Prediction Method for the correlation analysis for the redundancy check.

$\checkmark \quad$ Thou DICOM file is encrypted using the AES Encryption Algorithm for the transmission over a TCP/IP network.

$\checkmark$ Transmitted encoded DICOM file is received at the Decoder end and decoded to obtain the original DICOM file and the data associated with the same.

As in reference with the earlier models of ROI based techniques such as MAXSHIFT, EZW, ROI-VQ, does not require additional co-efficients to decode the image, here since we are implementing the compression algorithms using the Integer Multi Wavelet Transforms, waving off the necessity for the additional bits for encoding. Also, that the proposed algorithmic complexity is less and does not require additional over-head tasks. The ROI thus defined in this algorithm supports any arbitrary shape.

\section{Existing System Results}

The above figure shows the different stages that the algorithm execution procedure and the images that were produced upon the constraints. With the proposed system in this paper we will be able to achieve much better PSNR (Peak-Signal-to-Noise-Ratio), MSE (Mean Square Error) and CR (Compression Ratios), than the ones achieved through [1].

It is proposed that a much better performance than the results projected by the earlier proposed system [1]. The below figure also shows the analysis of the compression ratios with respect to the distortion levels associated with the images [1]. This analysis is carried since both the parametrics are necessary for PSNR and CR.

We also note that as the distortion level increases the CR and the PSNR parametric values decreases, thus we propose a system wherein these values will be visualized and monitored.

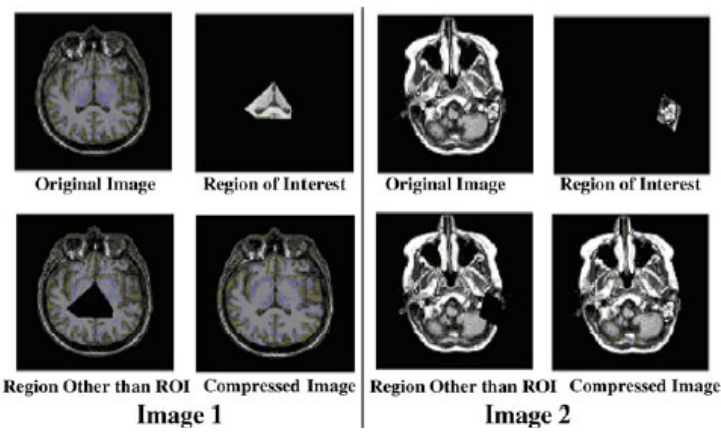

Fig1. Different Stages of the Image Developed in [1] 
Computer Applications: An International Journal (CAIJ), Vol.2, No.1, February 2015

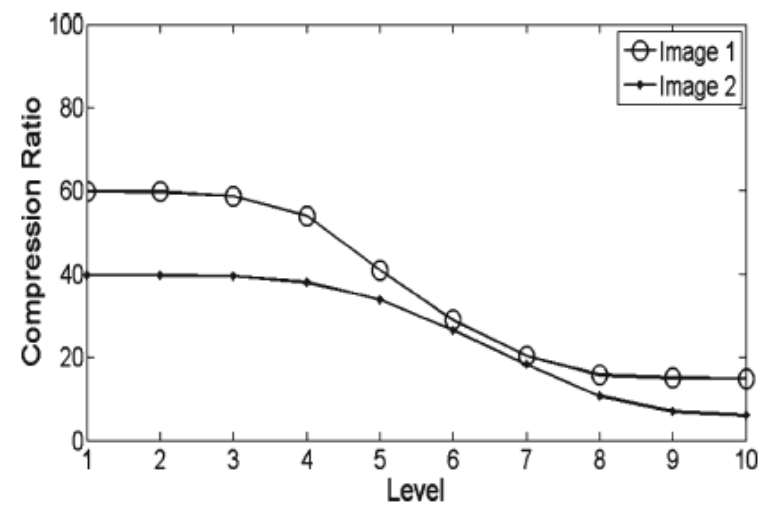

\begin{tabular}{lcccccccc}
\hline Sr. & Level & \multicolumn{3}{c}{ Image 1 } & & \multicolumn{3}{c}{ Image 2 } \\
No. & nmax & MSE & PSNR & CR & & MSE & PSNR & CR \\
\hline 1 & 0 & 0.0005 & 80.85 & 59.93 & & 0.0003 & 83.80 & 39.69 \\
2 & 1 & 0.0031 & 73.25 & 59.78 & & 0.0009 & 78.46 & 39.69 \\
3 & 2 & 0.0205 & 65.02 & 58.70 & & 0.0114 & 67.55 & 39.53 \\
4 & 3 & 0.2834 & 53.60 & 53.92 & & 0.1338 & 56.86 & 38.00 \\
5 & 4 & 2.3427 & 44.43 & 40.98 & & 0.8590 & 48.79 & 33.84 \\
6 & 5 & 17.2934 & 35.70 & 28.96 & & 3.8044 & 42.30 & 26.46 \\
7 & 6 & 48.3973 & 31.28 & 20.33 & & 20.8188 & 34.94 & 18.30 \\
8 & 7 & 357.641 & 22.59 & 15.71 & 36.3838 & 32.52 & 10.67 \\
9 & 8 & 704.679 & 19.65 & 14.93 & & 55.5300 & 30.68 & 6.98 \\
10 & 9 & 691.057 & 19.73 & 14.91 & & 153.323 & 26.32 & 6.04 \\
\hline & & & & & & &
\end{tabular}

\section{Conclusion}

Such a method is recommended for telemedicine system especially in rural area, where network resources have limitations. As a progressive approach to the recommended method, we may include much better performance metrics for compression, along with watermarking to maintain the authenticity of the medically produced images/data and reduce the various distortion levels associated with the image.

\section{Acknowledgment}

The authors would like to thank Management Panchajanya Vidya Peetha Welfare Trust (Regd), Dr. C. Nanjundaswamy our beloved Principal, Dr. R.Murali, Dr M V Mandi and Dr S Ramesh of Dr. Ambedkar Institute of Technology, Bengaluru for their assistance, suggestions, insight and valuable discussion over the course of this research work.

\section{References}

[1] Vinayak K Bairagi and Ashok M Sapkal, "ROI-based DICOM Image Compression Techniques", Sadhana, Vol.38, Part1, pp.123-131, February 2013

[2] Dr.E.Kannan and G.Murugan, "Lossless Image Compression Algorithm For Transmitting Over Low Bandwidth Line", ISSN: 2277128X, Vol2, Issue 2, February 2012

[3] Neha S Korde and Dr. A A Gurjar, "Wavelet Based Medical Image Compression For Telemedicine Application", American Journal of Engineering Research(AJER), e-ISSN:2320-0847, p-ISSN: 23200936, Vol.3, Issue-01, pp.06-111

[4] E.Praveen Kumar and Dr. M G Sumithra, "Medical Image Compression Using Integer Multi Wavelets Transform for Telemedicine Applications", Inernational Journal of Engineering and Computer Science, ISSN:2319-7242, Vol.2, Issue5, pp.1663-1669, May, 2013. 
Computer Applications: An International Journal (CAIJ), Vol.2, No.1, February 2015

[5] A Zscccarin and B Liu. "A novel approach for coding color quantized images", IEEE Trans, Image Processing, Vol.IP-2,pp.442-453, Oct. 1993

[6] D E Knuth, "Dynamic Huffman Coding”, J Algorithms, Vol.6, pp.163-180, 1985

[7] M J Weinberger, J Rissanen and R B Arps, "Appliations of Universal Context Modeling to Lossless Compression of gray-scale Images”, IEEE Trans, Image Processing, Vol. 5, pp. 575-586, Apr.1996 